\title{
Correction to: Impact of a mixed educational and semi-restrictive antimicrobial stewardship project in a large teaching hospital in Northern Italy
}

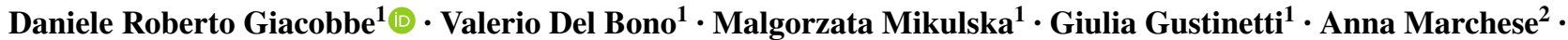 \\ Federica Mina ${ }^{3}$ - Alessio Signori ${ }^{4} \cdot$ Andrea Orsi $^{5} \cdot$ Fulvio Rudello $^{6} \cdot$ Cristiano Alicino $^{5} \cdot$ Beatrice Bonalumi $^{3}$. \\ Alessandra Morando ${ }^{7}$ - Giancarlo Icardi ${ }^{5} \cdot$ Sabrina Beltramini $^{3}$. Claudio Viscoli ${ }^{1}$ On behalf of the San Martino \\ Antimicrobial Stewardship Group
}

Published online: 5 October 2017

(c) Springer-Verlag GmbH Germany 2017

\section{Correction to: Infection}

DOI 10.1007/s15010-017-1063-7

A technical error led to incorrect rendering of the author group in this article. The correct authorship is as follows:

Daniele Roberto Giacobbe ${ }^{1}$, Valerio Del Bono ${ }^{1}$, Malgorzata Mikulska ${ }^{1}$, Giulia Gustinetti ${ }^{1}$, Anna Marchese ${ }^{2}$, Federica Mina ${ }^{3}$, Alessio Signori ${ }^{4}$, Andrea Orsi ${ }^{5}$, Fulvio Rudello $^{6}$, Cristiano Alicino ${ }^{5}$, Beatrice Bonalumi ${ }^{3}$, Alessandra Morando ${ }^{7}$, Giancarlo Icardi ${ }^{5}$, Sabrina Beltramini ${ }^{3}$, Claudio Viscoli ${ }^{1}$; On behalf of the San Martino Antimicrobial Stewardship Group

The online version of the original article can be found under doi:10.1007/s 15010-017-1063-7.

Daniele Roberto Giacobbe

daniele.roberto.giacobbe@edu.unige.it

1 U.O. Clinica Malattie Infettive, University of Genoa (DISSAL) and Ospedale Policlinico San Martino-IRCCS per L'Oncologia, L.go R. Benzi, 10, 16132 Genoa, Italy

2 S.S.D. Microbiologia, University of Genoa (DISC) and Ospedale Policlinico San Martino-IRCCS per L'Oncologia, Genoa, Italy

3 U.O. Farmacia, Ospedale Policlinico San Martino-IRCCS per l'Oncologia, Genoa, Italy

4 University of Genoa (DISSAL), Genoa, Italy

5 U.O. Igiene, University of Genoa (DISSAL) and Ospedale Policlinico San Martino-IRCCS per l'Oncologia, Genoa, Italy

6 SANTALUCIA PHARMA APPS $\odot$, Località Gragnanino, Gragnano Trebbiense, PC, Italy

7 U.O. Governo Clinico e Organizzazione Ospedaliera, Ospedale Policlinico San Martino-IRCCS per l'Oncologia, Genoa, Italy
The collaborators are:

Antonio Ferrazin, Ferdinando Dodi, Norberto Morandi, Federica Toscanini, Marco Camera, Antonio Di Biagio, Giovanni Mazzarello, Anna Ida Alessandrini, Andrea De Maria, Emanuele Delfino, Laura Ambra Nicolini, Carolina Saffioti, Federica Magnè, Nemo Gandolfo

The original publication has been corrected. 